\title{
Crystallography of Martensitic Transformation in Steels: Advances in Experimental and Theoretical Research
}

\author{
N.Yu. Zolotorevsky ${ }^{1}$ and A.A. Zisman ${ }^{1,2}$ \\ ${ }^{1}$ Institute of Applied Mathematics and Mechanics, Peter the Great Polytechnic University, St-Petersburg 195251, Russia \\ ${ }^{2}$ Central Research Institute of Structural Materials "Prometey”, St-Petersburg 191015, Russia
}

Received: March 05, 2020

\begin{abstract}
Advances in experimental and theoretical researches of martensite in steels are surveyed which attracts a renewed interest since EBSD and high resolution TEM techniques have provided new quantitative data on crystallography of martensitic transformations. Thus, inter-phase orientation relationships (ORs) can be determined by EBSD even in the absence of retained austenite. Such ORs, in turn, enable accurate analysis of <orientation> variant pairing which notably influence steel properties; the same data make it possible to reconstruct textures and hence evaluate structural states of prior austenite in terms of local orientations in martensite. Novel theoretical approaches aimed to overcome shortcomings of phenomenological theory of martensite crystallography were considered, including atom-scale topological models and the recently proposed micromechanical models. Finally, potentiality and limitations of the current advances are discussed.
\end{abstract}

\section{INTRODUCTION}

Although steels could hardly be regarded as "advanced materials", they attract persisting attention displayed by thousands of publications appearing every year. Such an interest to steels is obviously due to ability of the $\mathrm{Fe}$ $\mathrm{C}$ system to provide a wide variety of structural states by means of thermo-mechanical treatments, even at a fixed chemical composition. Of course, this variety greatly multiplies with allowance for the effects of alloying [1,2].

An important prerequisite of special attention currently given to the crystallography of steel microstructure is the appearance of electron backscatter diffraction (EBSD) technique. Unlike the previous approach, where mechanical properties were related to microstructure parameters (dimensions and shapes of grains, laths or packets and the dislocation density), EBSD has revealed the significant effect of crystallographic factors. It has been shown that local crystal orientations or/and misorientations depending on the processing mode notably affect the steel properties; in particular, a high fraction of high angle boundaries provides effective barriers to cleavage fracture [3-12].
In the present review, we consider the microstructures produced in steels by displacive phase transformations; products of the latter are referred to as martensite or bainite. The lath martensite that is formed in low and medium carbon steels will be considered in what follows. Although the origination of bainite is under discussion up to date [13], the authors believe that its nucleation and growth mostly proceed by the displacive mechanism. Apparently, diffusional effects do matter on the later stage of bainitic transformation; however, they cannot notably alter lattice orientation of growing crystallites.

\section{CURRENT STATE OF CRYSTALLOGRAPHIC ANALYSIS}

\subsection{General characterization of martensite/bainite constitution}

Crystallography of the considered steel microstructures formed by the phase transformation in cooling is predetermined by an orientation relationship (OR) between the crystal lattice of each parent grain of austenite $(\gamma$ phase) and the products ( $\alpha$-phase crystals) within it. In

Corresponding author: N.Yu.Zolotorevsky, e-mail: zolotorevskii@mail.ru 

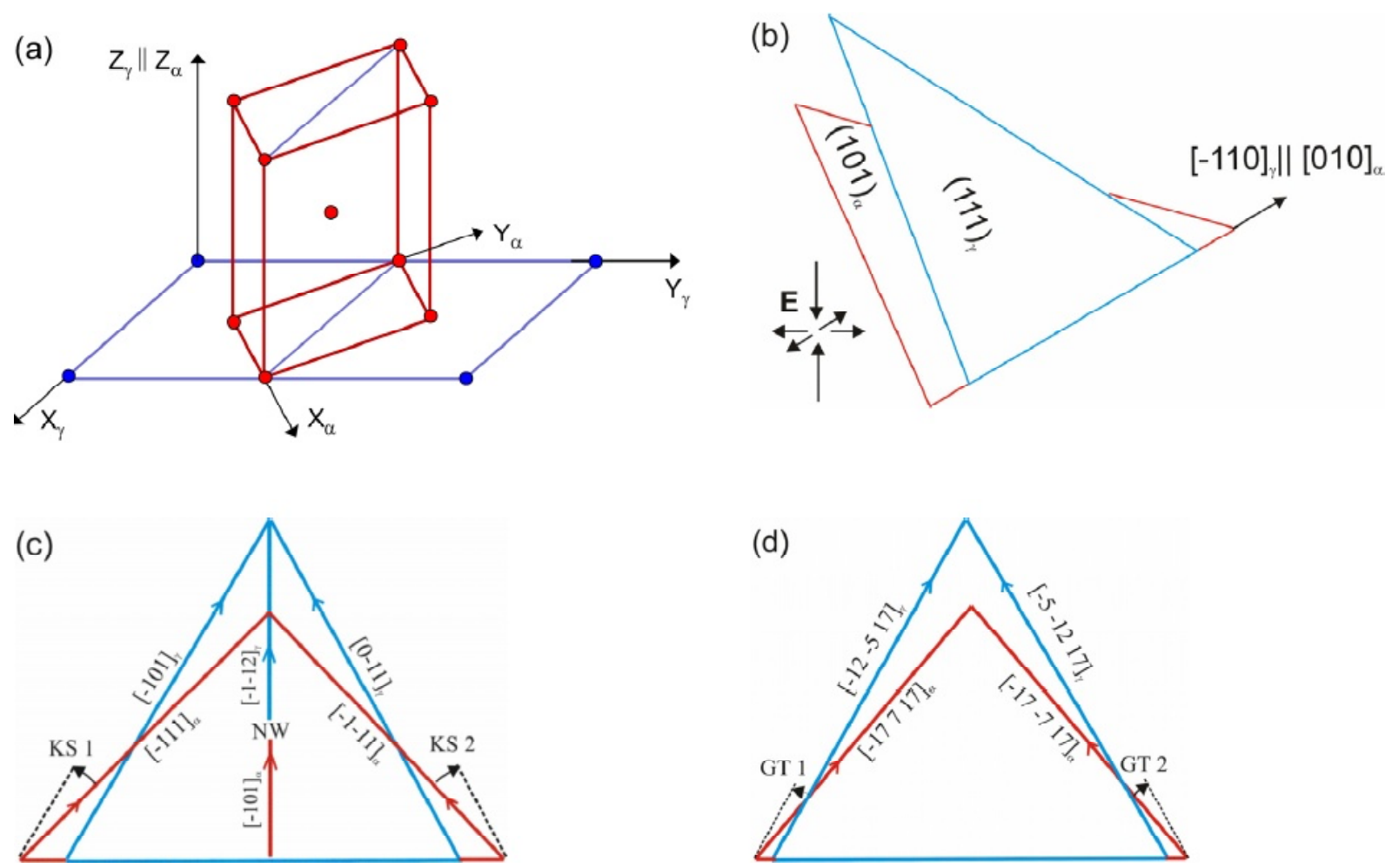

Fig. 1. The Bain scheme of lattice transformation: (a) a virtual embryo of elementary bcc cell in the parent fcc crystal; (b) relative rotation and strain of nascent plane $(101)_{\alpha}$ due to the transformation strain ("Bain strain"); directions which should align to each other in $(111)_{\gamma} \|(101)_{\alpha}$ according to orientation relationships: (c) KS or NW and (d) GT.

low carbon martensite and bainite of the lath morphology the OR is usually close to Kurdjumow-Sachs (KS) one [14], whereas in high carbon or severely alloyed steels it is closer to the Nishiyama-Wasserman (NW) one $[15,16]$ rotated from $\mathrm{KS}$ by $5.26^{\circ}$. Both ORs require parallelism of the close packed $\{111\}_{\gamma}$ and $\{110\}_{\alpha}$ planes (CPPs); at that, parallel close packed directions (CPDs) are $\langle 110\rangle_{\gamma} /\langle 111\rangle_{\alpha}$ in the case of KS OR and $\langle 112\rangle_{\gamma} /$ $\langle 011\rangle_{\alpha}$ in the case of NW OR. In general, most of real ORs are intermediate between KS and NW [17-23], often close to Greninger-Troyano (GT) relationship [24]. The reference OR are shown in terms of the lattice transformation where, according to Bain [25], virtual BCC cells of the product are easy to recognize in the parent FCC lattice, Fig. 1a. Virtual rotation and strain of the nascent martensite plane $(101)_{\alpha}$ with respect to initial (111) $)_{\gamma}$ are illustrated in Fig. 1b whereas alignment of certain directions according to above mentioned ORs in eventually parallel planes (101) and (111) $)_{\gamma}$ are shown in Figs. 1c and 1d.

Owing to the symmetry of cubic lattice, any near-KS OR predetermines 24 orientation variants that may develop from a single parent grain, provided annealing twins are disregarded. Following a list proposed in Ref. [17], the variants constituting this set are usually called V1 to V24, where V1 is arbitrary selected. A microstructural unit corresponding to a single orientation variant is referred to as block; on a finer scale a martensite block consists of several slightly (by few degrees) disoriented "laths" [18], which belongs to the same crystallographic variant. In the case of bainite, blocks have somewhat more complex substructure; in particular, its laths may contain smaller subunits [19]. On a coarser scale, the blocks are grouped into packets where laths have a nearly unique habit plane corresponding to the parallelism of the same close-packed planes of the two phases. Therefore, the microstructure inside a former grain has threelevel hierarchy: packets, blocks, and laths.

The above-mentioned dependence of the martensite/ bainite crystallographic parameters on the processing conditions is mostly associated with variant grouping (paring), the phenomenon studied extensively by EBSD analysis in the last two decades [3,5,11,12,20-23,26-31]. It turned out that variant selection during phase transformation occurs so that certain orientations predominantly appear side by side. For instance, in the case of lath martensite, the variants with a low misorientation angle, V1/V4, form pairs within a single packet. That is why the crystallites consisting of such alternative variants sometimes are also called martensite blocks, while a unique variant is treated as sub-block $[17,18,23]$. Unlike martensite, in bainite formed at a relatively low temperature, close to the martensite start, high-angle boundaries V1/V2 predominate within a packet [11,12,22]. 

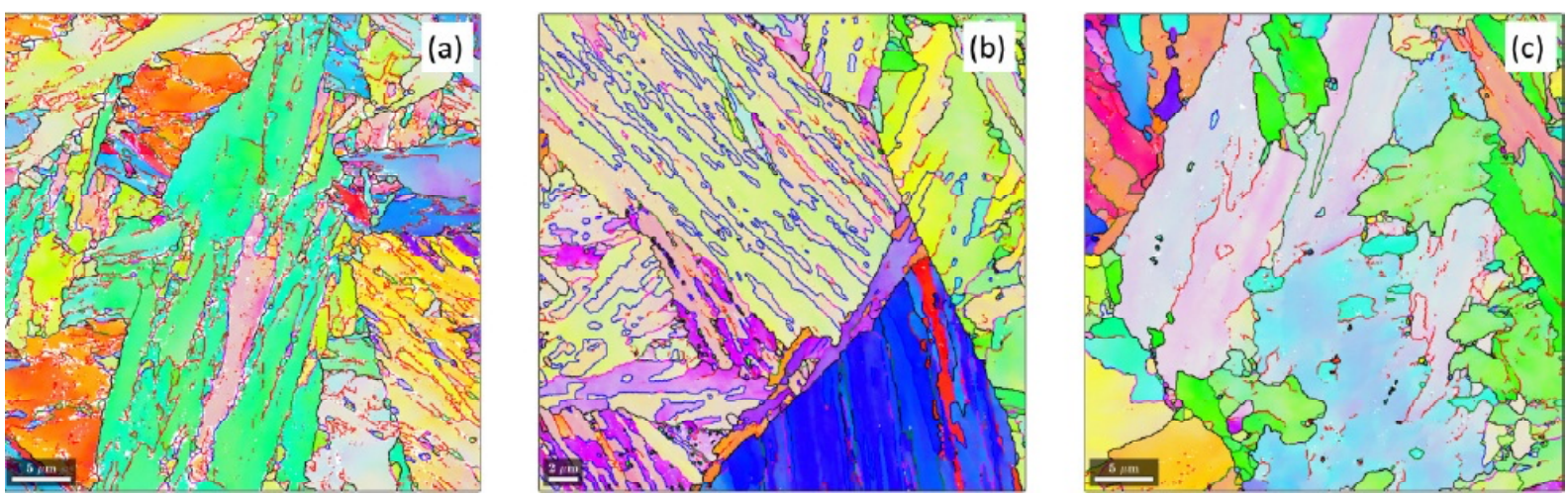

Fig. 2. Microstructures of three low carbon steels represented using inverse pole figure maps with superimposed boundaries of certain types: high angle random boundaries (black), V1/V4 (red), V1/V2 (blue), V1/V3 \& V1/V5 (magenta) and V1/V8 (green). a-martensite; b-lower temperature bainite, $\mathrm{c}$ - higher temperature bainite.
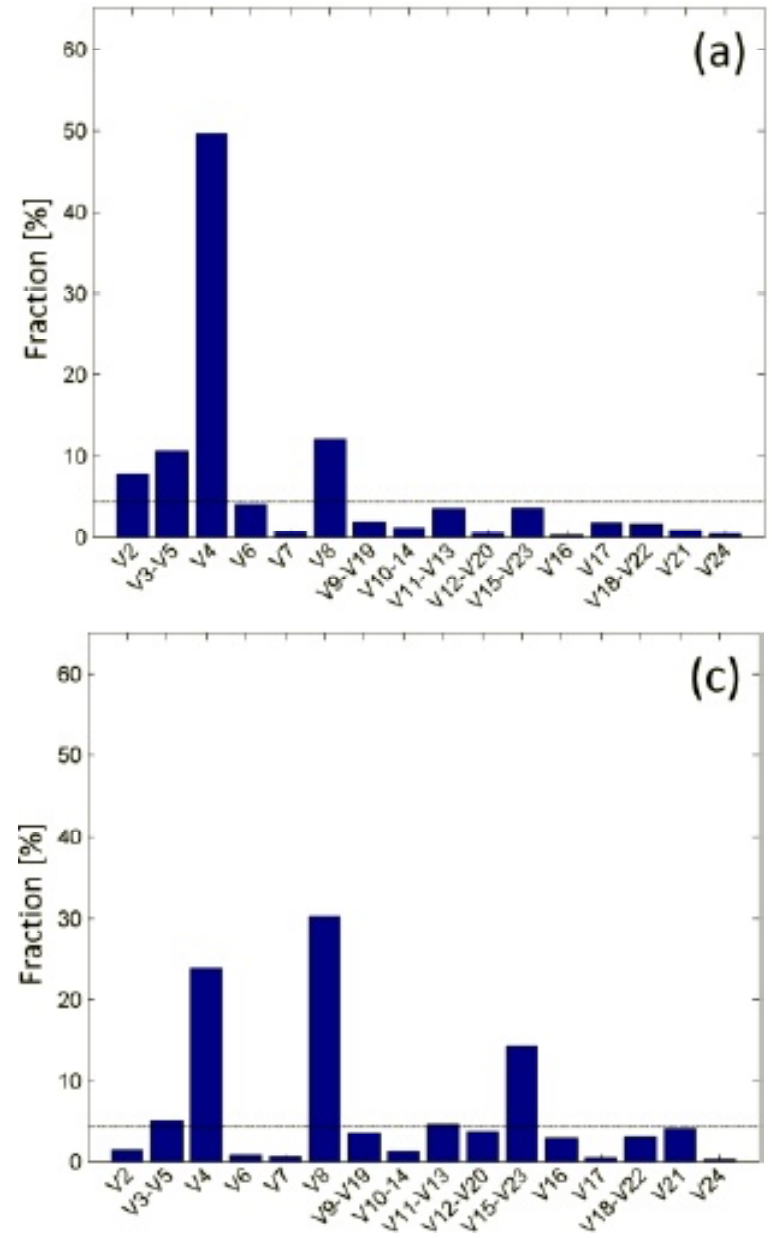

When increasing the transformation temperature, the fraction of V1/V2 boundaries decreases, the packet structure degenerates and low-angle inter-variant boundaries V1/V8 become typical [29, 31]. It should be noted that in case of the plate martensite, which is found in high-carbon steels, another pattern of variant paring is observed where structural hierarchy peculiar to the lath martensite is no longer evident [26]. Examples of the martensite/bainite microstructures and corresponding length fractions of inter-variant boundaries are shown in Figs. 2 and 3, respectively.

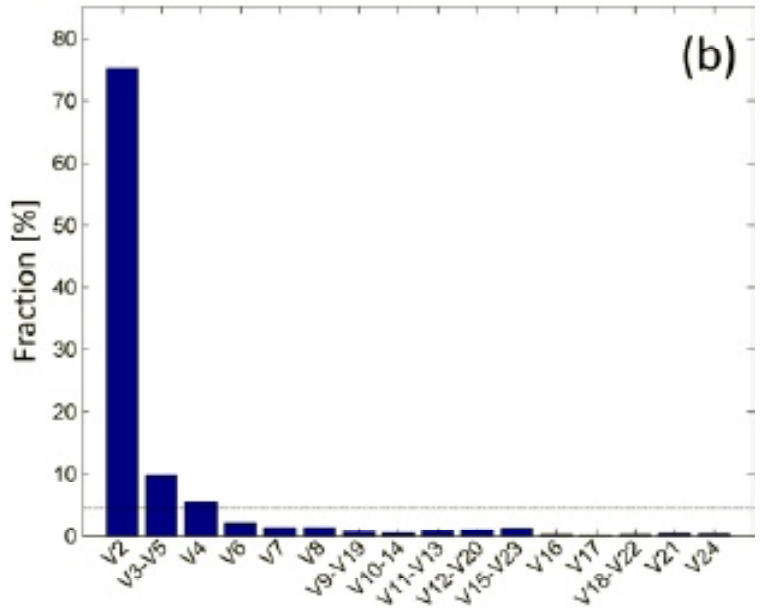

Fig. 3. Length fractions of inter-variant boundaries for the steels, whose microstructure is shown in Fig. 1. amartensite; $\mathrm{b}$ - lower temperature bainite, $\mathrm{c}$ - higher temperature bainite.

The variant paring has been often considered $[4,11,32]$ to minimize specific energy of inter-variant boundaries and that due to residual stresses. To diminish the latter, not only plastic shearing in martensite or austenite but also self-accommodation of lath packets is considered. When controlling the fraction of highangle boundaries and hence an effective size of structural elements in order to improve the steel properties, a quantitative crystallographic analysis of microstructure is an indispensable means. Besides, it would be of great practical significance to reconstruct a structural state of 
the parent austenite from the transformed structure; this is possible owing to pronounced OR between the parent and product phases of martensitic or bainitic steel. At the same time, precision of both the variant paring characterization and the austenite structure reconstruction strongly depends on accuracy of the employed OR [26]. It is also worth noting that determination of actual ORs is the most direct way to verify theoretical models of martensitic transformation. Accordingly, efforts of many scientists are currently focused on the development of relevant techniques [27,32-45].

Owing to appearance of various novel techniques, complex structures of martensite can be simultaneously analyzed in $3 \mathrm{D}$ at various scales that provides previously unavailable important data. In case of low carbon martensite, for example, performance of such an approach is demonstrated in [46].

\subsection{Determination of OR between parent and product phases}

The KS OR is usually considered as a reference OR in steels though some violation of parallelism presumed for both the CPPs and CPDs has long been known. That is why, for ease of interpretation various real OR are often specified by angular deviations of corresponding CPPs and CPDs. Before the EBSD technique has appeared, studies of the OR for martensitic and bainitic transformations were mostly based on TEM applied to local areas containing islands of retained austenite. However, desired accuracy was difficult to achieve because of low quantity of retained austenite and/or high density of dislocations in the transformed crystals [32]; besides, the employed local method is hardly significant statistically. Moreover, orientations determined in thin inter-lath layers of retained austenite differ from those of former austenite grains because of plastic accommodation in both phases during the transformation. In order to improve determination of OR, convergent beam Kikuchi line diffraction pattern analysis in TEM [32] was used. This method reduces the effect of the high dislocation density; however, there remain other limitations mentioned above. In Ref. [32], the same ORs has been determined in lath martensite and bainite: $\{111\}_{\gamma} \|\{110\}_{\alpha},\langle 110\rangle_{\gamma}$ deviate from $\langle 111\rangle_{\alpha}$ by $2.5^{\circ} \pm 2^{\circ}$. One can see that the CPD scatter is rather large.

The EBSD orientation mapping in steel microstructures notably enhanced statistical significance of OR determination. Furthermore, a principal advantage of this approach is that it works regardless of quantity and even presence of retained austenite [27,33-44]. Most of related methods use orientations of $\alpha$-phase variants formed within an individual parent austenite grain in order to determine simultaneously the orientation of the austenite grain and the OR between the austenite and the product phase. All of them solve this problem with more or less high accuracy when parent austenite had been recrystallized prior to phase transformation. However, due to the orientation non-uniformity in parent austenite grains, it is difficult to apply such methods to ausformed steels. Apart from earlier efforts to get over this difficulty [36,37], novel approaches based on intervariant misorientations have been developed [41-45] which do not require reconstruction of parent grains. According to Ref. [41], ORs in recrystallized and plastically deformed states of austenite are similar within the statistical dispersion. Owing to simplicity of this method, it has found applications to microstructures formed by various thermo-mechanical treatments [12].

OR derived from EBSD data on lath martensite of low carbon steels [22, 23,27,36,38-43,45] showed that inter-phase angular deviations of CPPs and CPDs gen-

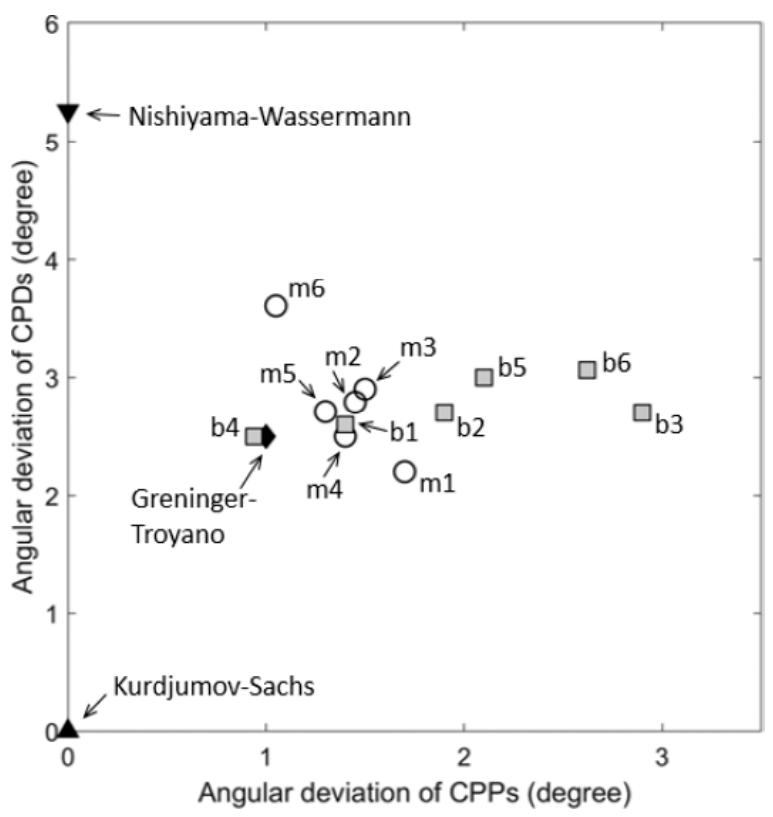

Fig. 4. Angular deviations of CPPs and CPDs for the orientation relationships obtained by various researchers using EBSD technique in martensite (m1-m6) and bainite (b1-b6) of several low-carbon steels, for which a carbon content in weight \% is indicated (additionally, the temperature of isothermal holding is indicated for b1-b3, while approximate mean temperature of transformation is indicated in the case of continuous cooling for b4-b6): $\mathrm{m} 1-0.05 \mathrm{C}$ [43], $\mathrm{m} 2-0.085 \mathrm{C}$, severely alloyed [42], m3-0.15C [21], m4 - 0.15C [39], m5-0.2C [41], m6 -0.2C, severely alloyed [44], b1 $-450^{\circ} \mathrm{C}[21]$, b2-500 ${ }^{\circ} \mathrm{C}[21], \mathrm{b} 3-580^{\circ} \mathrm{C}[21], \mathrm{b} 4-\sim 430^{\circ} \mathrm{C}[21], \mathrm{b} 5-$ $\sim 470^{\circ} \mathrm{C}[21]$, b6 $-\sim 520^{\circ} \mathrm{C}$ [21]. Reference orientation relationships of Kurdjumov-Sachs, Nishiyama-Wassermann and Greninger-Troyano are also shown on the plot. 
erally vary in the ranges $\sim 0.5-2.0$ and $\sim 1.5-3.5$ degrees, respectively, depending on steel composition. Some results found in the literature are represented in Fig. 4. One can see that most of them appear to be rather close, varying by about $0.5^{\circ}$ from the GT OR. In case of bainite, some data for which are also represented in Fig. 4, the CPP deviation remains close to that of martensite, while the $C P D$ deviation varies within a wider range, from $\sim 1^{\circ}$ to $\sim 3^{\circ}$ with increasing temperature of transformation. Furthermore, OR scatters around the mean (the most probable) positions by a few degrees [37]. This variation has been ascribed not only to measurement inaccuracy but also to non-uniformity of real OR within its variants $[37,44]$.

Unlike the conventional phenomenological theory of martensite crystallography (PTMC) [39-49] that allows for plastic shearing only in crystals of the nascent phase, Miyamoto et al. [50] have shown that plastic accommodation in austenite also takes place and hence affects the inter-phase OR. Apart from respective PTMC correction, this effect is considered to explain actual OR variations, both those of mean OR and the scatter of OR observed in the same steel [37,51]. Relevance of such a viewpoint is supported, in particular, by the temperature dependence of experimental OR in bainite [21]. Although the previous speculations are qualitative, plastic accommodation in austenite undoubtedly influences crystallography of martensite and bainite transformation and hence should be integrated in rigorous models.

\subsection{Atomic-scale constitution of martensite habit planes}

According to high resolution TEM data, near-planar boundaries between martensite and austenite actually have terrace-like constitution where microscopic $\{111\}_{\gamma} \|\{110\}_{\alpha}$ facets are separated by monoatomic steps. Thus, habit planes of laths thus appearing in a $\mathrm{Fe}-\mathrm{Ni}$ Mn alloy vary around $\{112\}_{\gamma}$ [52]; earlier investigations of such alloys [53] revealed habit planes close to $\{557\}_{\gamma}$ although the regular TEM could not resolve their stepped structure. Besides, average habits of terrace-like boundaries close to $\{225\}_{\gamma}$ have been detected in a high carbon plate martensite alloyed by $8 \mathrm{wt}$. \% of $\mathrm{Cr}$ [54]. On the one hand, these findings explain the quick martensite growth by easy movement of the steps along the coherent plane; on the other hand, they admit a reach variety of known martensite habits by means of the length variation of short (several atoms) $\{111\}_{\gamma}$ constituents. Note that PTMC could not explain habit planes $\{557\}_{\gamma}$ and $\{225\}_{\gamma}$ formed in this way.

As discussed in Subsection 3.3, the mentioned atomscale data proved to be an important prerequisite to develop so called topological models of martensite which partly avoid drawbacks of PTMC. At the same time, these essentially local models have own limitations which hopefully can be eliminated in terms of PMTC or the micromechanical approach considered in Subsection 3.2 .

\section{MODELS FOR MARTENSITE CRYSTALLOGRAPHY}

Since the models based on the original PTMC are surveyed elsewhere [55-57], we will only briefly note shortcomings of this approach and then focus on its later extensions. According to PMTC, slip or twinning systems of a nascent martensite crystal should be indicated first to accommodate the misfit of its transformation strain with the parent (austenite) phase. After one or two trial systems are a priori selected, respective plastic shears are determined to satisfy the condition of invariant plane strain (IPS) i.e. no strain misfit at some planar interface further considered for the martensite habit. The corresponding apparent shear along this plane and the interphase OR are then compared to relevant experimental data. Such a hit-and-miss approach to the actual shearing scheme eventually explains formation of various final structures; however the latter and, moreover, their dependence on transformation conditions cannot be unambiguously predicted in this way. Indeed, as widely accepted, crystallography of martentsite is preset by the transformation strain and plastic shearing of the product, but PTMC solves an inverse problem where accommodative shear systems are sought among admitted candidates to comply with the known structure.

\subsection{Extensions of PTMC}

Development of double shear models for plastic accommodation of transformed crystals [58] and its applications to various martensitic and bainitic steels $[4,59,60]$ provided an important advance of PTMC previously unable to explain some habit planes such as $\{225\}_{\gamma}$ and $\{557\}_{\gamma}$. Besides, such models have enabled deeper insight in constitution of a martensite lath or plate where specific single shears may develop in separated domains [61] thus resulting in their dissimilar orientations. It is worth noting that allowance for the second plastic shear was algebraically inevitable since the $2 \mathrm{D}$ tensor of strain misfit at any planar interface has two scalar invariants and hence necessitates at least two degrees of freedom in its accommodation. However, regardless of enhanced understanding, the considered extension of the original theory still inherits its prediction uncertainty as far as no independent criterion is applied to indicate a unique shearing scheme.

In the last decade, several efforts have been undertaken to employ the double shearing scheme while as- 
cribing constitutive shears to the lattice transformation itself rather than following plastic accommodation of the transformed crystal. There is a crystallographic prerequisite for similar models since disorientation between some variants of KS OR corresponds to the twinning. Thus, according to [62], a lath with habit plane $\{557\}_{\gamma}$ can be composed of two such variants occupying similar volume fractions. However, twin disorientations, ascribed to whether transformation or plastic accommodation, can hardly appear in low carbon martensite where laths generally contain a high dislocation density evidencing for activity of slip systems rather than twinning. At the same time, this remark is irrelevant to higher carbon steels where the twined constitution of plates is observed that admits both a specific transformation mode and accommodation by deformation twinning. Although additional data were necessary to justify one of these schemes, the former alternative has been presumed [63] to compose a $\{225\}_{\gamma}$ plate of twin-related KS variants. Later, the same authors [64] reconsidered habit plane $\{557\}_{\gamma}$ of low carbon martensite by combination of low disoriented of KS variants in a single lath. Using this OR for the only input data, this model is based on a specific concept of angular compatibility rather than the conventional IPS requirement ensuring no strain misfit on inter-phase boundaries. As to phase stresses, thus ignored, they have been assumed to vanish by means of plastic accommodation in austenite surroundings according to [50]. However, to get a self-consistent model, this qualitative reasoning should be embodied in a rigorous form.

Apart from disoriented domains of a single lath or plate collectively diminishing the average strain misfit at its habit plane, self-accommodation of lath packets [60] is also considered when the plastic accommodation is inefficient for some reason, e.g. low temperature. In this case, to avoid accumulation of the net shear deformation within the packet and hence the growing stress in its interaction with surroundings, directions of transformation shears in parallel laths should notably alternate; in other words, respective OR variants should correspond to different Bain axes. However, quantitative models for inter-lath stress sources are still wanted to predict constitution of whole packets.

Unlike an algebraic formulation of PTMC treating all at once effects of the transformation strain and plastic accommodation, some recent models try to follow the development of these phenomena. Thus, an effort is undertaken to combine with PTMC the incremental modeling of finite accommodative shears [65]. Although active slip systems still could not be uniquely selected in this way, such an approach peculiar to the conventional crystal plasticity enables allowance for temperature, the strain rate and chemical composition of steel. Accordingly, the cooling conditions gain in significance since they affect time and hence the rate of accommodative shearing as well the heat release and temperature stagnation at the initial transformation stage. Moreover, such implications complying with experimental data [66] suggest the requirement of ideal IPS (no strain misfit at planar interfaces) to weaken. Indeed, the plastic shearing by any slip system of martensite stops at some critical level of the resolved shear stress and hence at a finite residual stress proportional to the inter-phase strain misfit.

\subsection{Micromechanical modeling of martensite nucleation}

Motivated by the famous model by Eshelby [67] and the previous reasoning, a micromechanical model of an embryonic martensite lath [68] has recently been formulated to discard uncertainty of PTMC while saving with appropriate accuracy its geometrical fundamentals. They are the IPS concept and the Bain scheme [25] of lattice transformation. Specifically allowing for constraint of a freshly transformed crystal by austenite, this model unambiguously selects accommodative slip systems and thus determines lattice reorientations during the plastic shearing. When applied to Bain axis $[001]_{\mathrm{g}}$ at habit plane (557) $)_{\gamma}$ or (111) $)_{\gamma}$ peculiar to low carbon martensite, the model predicts simultaneous activity of two slip systems as could be expected since two indices of this plane are equivalent with respect to the considered transformation strain. At the same Bain axis and alternative habit plane $(755)_{\gamma}$ the situation is no longer symmetric and, following the conventional PTMC scheme, suggests a sequence of simple shears by dissimilar slip systems. However, unlike PTMC where such a scheme is sought by the hit-and-miss method, both the desired systems and the transition shear are uniquely predicted.

Fig. 5 compiled from [68] illustrates performance of the micromechanical model as applied to habit plane (755). The first slip system is selected to provide the quickest diminishing of interfacial strain misfit $s$ initially induced by the lattice transformation. However, the lattice rotation by the increasing plastic shear $\gamma$ gradually reduces efficiency $\xi=d s^{2} / d \gamma$ of this system that is eventually substituted by another one (Fig. 5a). According to Fig. 5b, the martensite crystal approaches $s^{2}=0$ (IPS state) within a reasonably small error. Moreover, small inter-phase deviations of CPPs and CPDs (Figs. 5c and $5 \mathrm{~d})$ indicate that that the resulting martensite orientation is very close to the KS OR.

\subsection{Topological approach to atom-scale constitution of interphase boundaries}

With allowance for the high resolution TEM data mentioned in Subsection 2.3, another alternative approach 

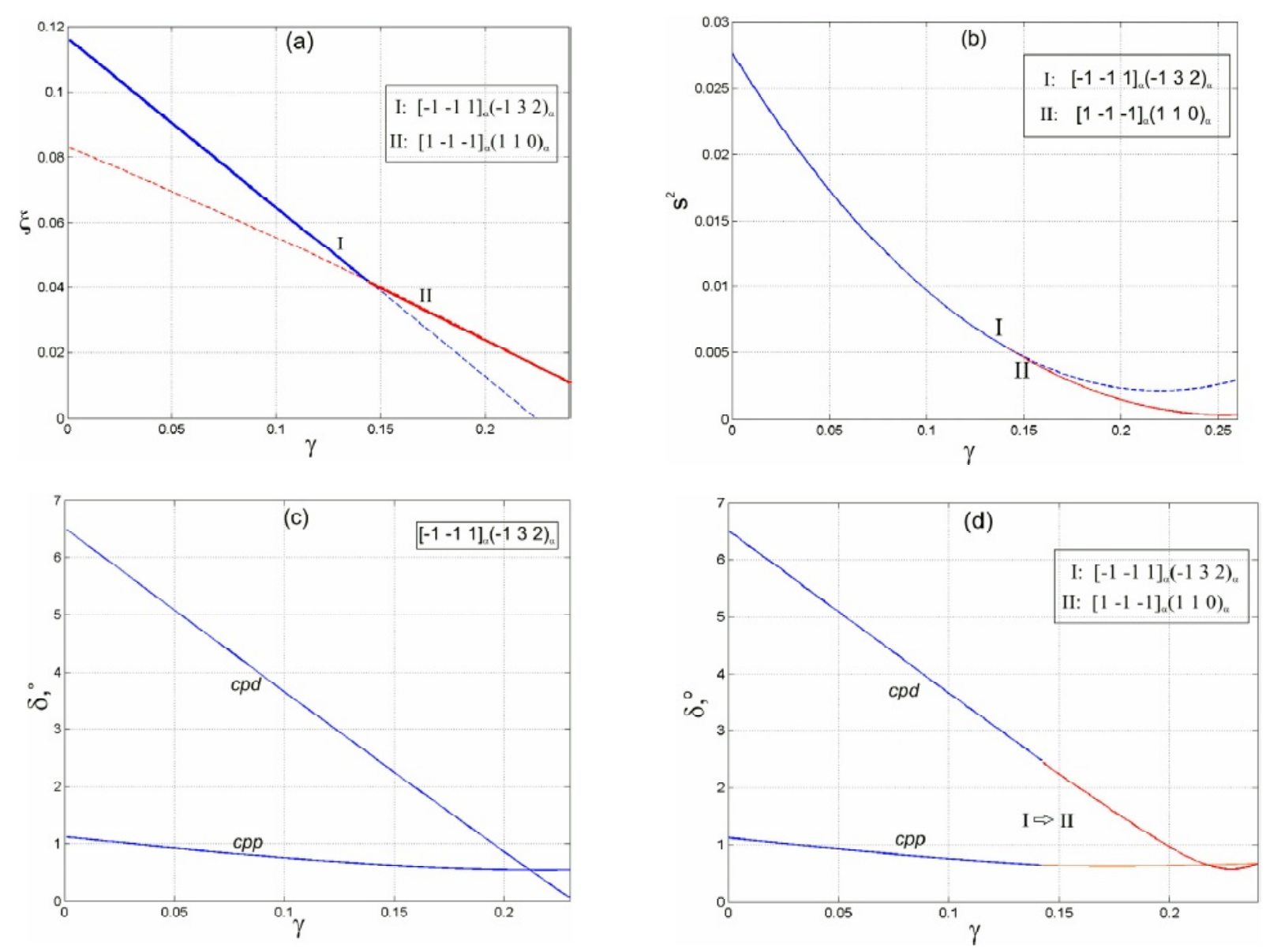

Fig. 5. Plastic accommodation simulated in [68] for a crystal transformed with Bain axis [001] at habit plane (755): (a) transition from the first to the second slip system in terms of their efficiency, (b) reductions of quadric measure for inter-phase strain misfit by single shears in one (upper) and two (lower) steps, (c, d) respective deviations of CPPs and CPDs.

to crystallography of martensite has been developed [69-71]. Related "topological models" (TM) correspond to the terrace-like constitution of inter-phase boundaries on the atomic scale and thus explain various known combinations of OR and habit planes. Specifically, when a certain habit (ratio of the step height to the length of terrace elements) is considered, the plastic shearing scheme of martensite is determined to minimize incoherence between dissimilar lattices across the interface. Hence, like the micromechanical model [68], this approach eliminates uncertainty in selection of slip systems peculiar to PTMC. Amazing, thus admitted combinations of habit planes and OR often prove to be similar to those explained by PTMC although the latter ignores the atom-scale constitution of interphase boundaries and aims to exclude the interfacial strain misfit and related stresses rather than incoherency of coexisting lattices. Apparently, the above-mentioned paradox remaining a subject of discussions [72,73] has a simple geometrical origin since the same lattice transformation induces both the mechanistic strain incompatibility con- sidered in PTMC and atom-scale coherence strains treated by TM.

It is worth noting that verification of TM approach by TEM data was not unblemished. Indeed, spacing of 3-6 $\mathrm{nm}$ between interfacial dislocations revealed in lath martensite of Fe-Ni-Mn alloys [53] notably exceeds a typical $\{111\}_{\gamma}$ facet of about $1 \mathrm{~nm}$ in length [52]. In other words, unlike the considered models [69-71], representative areas of the dislocation networks can hardly fit in single facets separated by monoatomic steps. This seems to be a good reason to treat such dislocations in usual mechanistic terms of PTMC rather than an atomscale approach. The authors could not find HR TEM data on terrace-like boundaries in lath martensite of low carbon steels; however, its dislocation density of the order of $10^{11} \mathrm{~cm}^{-2}$ seemingly supports the previous conclusion as far as the related dislocation spacing and, accordingly, the distance between active slip planes are not less than $10 \mathrm{~nm}$.

Despite advantages of TM, this approach cannot uniquely predict a habit plane and hence should em- 
ploy it for an input parameter. Meanwhile, according to the micromechanical model [68], the habit plane is preset by the transformation strain before plastic accommodation starts although additional physical data are needful for precise predictions. To this end, for example, microscopic estimates of specific energy of semi-coherent boundaries [71] could be combined with the mechanistic treatment of transformation strain misfit at apparent habit planes. Interesting, such an analysis will suggest that a growing embryonic lath gradually deviates from its initial coherent plane $\{111\}_{\gamma}$ despite some increase of interfacial energy. Indeed, while the constrained nascent crystal enlarges, its bulk elastic energy undoubtedly gains in significance with respect to the coherence issue. Although this reasoning wants a rigorous quantitative formulation, it finds a significant support in the above-mentioned high resolution TEM data [52,54]. Really, apparent habit planes of the detected terrace-like boundaries of martensite crystals mostly vary around $\{112\}_{\gamma}$ corresponding to the least incompatibility of the lattice transformation strain as evaluated in [68]. Moreover, according to the latter, a drift to $\{557\}_{\gamma}$ and then $\{112\}_{\gamma}$ from embryonic habit planes $\{111\}_{\gamma}$, peculiar to stacking faults of austenite, provides a gradual reduction of the interfacial strain misfit and hence related phase stresses.

\section{SUMMARY}

Considerable progress has been made in the study of martensitic transformation in steels over the last two decades, both in the experimental investigations and in the theory of martensite crystallography.

With respect to experimental achievements, the phenomenon of variant grouping (paring) is of particular interest. The character of paring was shown to differ significantly in martensite and in bainites of various kinds, and this allows to distinguish different structural constituents even when their morphology is similar. The variant paring controls the fraction of high-angle boundaries and hence an effective grain size, and thereby enables one to affect steel properties by variation of processing mode. A success has been achieved also in the reconstruction of the parent austenite from the transformed structure, which allowed shedding light on the effect of austenite condition on the phase transformation.

Precision of both the variant paring characterization and the austenite reconstruction strongly depends on the accuracy of employed OR. The EBSD orientation mapping enabled to determine the OR with a high precision based only on the orientations of crystallographic variants in the product phase without residual prior austenite. Different EBSD methods developed to determine OR give similar results, which confirms their reliability.

Another fundamental crystallographic characteristics is a habit plane. The terrace-like constitution of boundaries between the parent and the product phases on the atomic scale has been established using high resolution TEM. These findings, on the one hand, explain the quick martensite growth by easy movement of steps along the coherent plane and, on the other hand, admit a reach variety of known martensite habits including those, which cannot be explained by PTMC.

Theoretical efforts were directed to overcome shortcomings of PTMC and to better explain observed crystallographic features of the transformed microstructure. Though double shear models suggested for plastic accommodation of transformed crystals in the frames of PTMC provided explanation of main crystallographic features including habit planes, they still inherit the prediction uncertainty of PTMC. Several efforts have been undertaken to implement the double shearing scheme to the lattice transformation itself rather than to the following plastic accommodation. However, such models either employ twinning as a shear mode, which is not actual for low carbon martensite and bainite, or are lacking quantification.

Alternative way to improve theory was to develop a micromechanical model of an embryonic martensite lath in order to discard uncertainty of PTMC while saving its geometrical fundamentals. Allowing for constraint of a freshly transformed crystal by austenite, this model unambiguously selects accommodative slip systems and thus determines lattice reorientations during the plastic shearing. Another alternative approach to crystallography of martensite has been developed with allowance for TEM observation of the terrace-like constitution of inter-phase boundaries. Like the micromechanical model, this approach eliminates uncertainty in selection of accommodative slip systems. Advantages of this approach as well as its shortcomings are discussed in the review.

Note in conclusion, that considerable plastic shears and lattice rotations in austenite have been observed near the transformation front. Thus, in addition to plastic accommodation inside the product phase, which was accounted for in the above considered models, the plastic accommodation in the parent phase should be included in consideration in order to develop a self-consistent quantitative theory. One more step towards the comprehensive theory of martensite crystallography is the consideration of self-accommodation, which was suggested to occur on a relatively large scale of lath packets. 


\section{REFERENCES}

[1] G. Krauss, Steels heat treatment and processing principles (Ohio, ASM International. 1990).

[2] R.W. K. Honeycombe, Steels, Microstructure and Properties (Edward Arnold, London, 1981).

[3] A.-F. Gourgues, H. M. Flower, and T. C. Lindley, Electron backscattering diffraction study of acicular ferrite, bainite, and martensite steel microstructures, Mater. Sci. and Technol., 2000, vol. 16, no. 1, pp. 26-40. https://doi.org/10.1179/ 026708300773002636.

[4] A. Lambert-Perlade, A.F. Gourgues, and A. Pineau, Austenite to bainite phase transformation in the heat-affected zone of a high strength low alloy steel, Acta Mater., 2004, vol. 52, no. 8, pp. 2337 2348. https://doi.org/10.1016/j.actamat.2004.01.025

[5] A. Lambert-Perlade, A.F. Gourgues, J. Besson, T. Sturel, and A. Pineau, Mechanisms and Modeling of Cleavage Fracture in Simulated Heat-Affected Zone Microstructures of a HighStrength Low Alloy Steel, Metal . Mater. Trans. A, 2004, vol. 35A pp. 1039-1053. https://doi.org/ 10.1007/s11661-004-0030-y

[6] Z. Guo, C.S. Lee and J.W. Morris Jr., On coherent transformations in steel, Acta Materialia, 2004, vol. 52, no. 19, pp. 5511-5518. https://doi.org/ 10.1016/j.actamat.2004.08.011

[7] S. Morito, H. Yoshida, T. Maki, X. Huang, Effect of block size on the strength of lath martensite in low carbon steels Mater., Sci. and Eng. A, 2006, vol.438-440, pp. 237-240. https://doi.org/10.1016/ j.msea.2005.12.048

[8] H. Kitahara, R. Ueji, N. Tsuji, Y. Minamino, Crystallographic features of lath martensite in low-carbon steel, Acta Mater., 2006, vol.54, no. 5, pp. 1279-1288. https://doi.org/10.1016/j.actamat. 2005.11.001

[9] H.Terasaki, Y. Miyahara, K. Hayashi, K. Moriguchi, and S. Morito, Digital identification scheme for steel microstructures in low-carbon steel, Mater. Characterization, 2017, vol. 129, pp. 305-312. http:// dx.doi.org/10.1016/j.matchar.2017.05.021

[10] N. Zolotorevsky, E. Kazakova, A. Kazakov, S. Petrov, and S. Panpurin, Investigation of the Origin of Coarse-Grained Bainite in X70 Pipeline Steels by EBSD Technique, Materials Performance and Characterization, 2017, vol. 6, No 3, pp. 281-291. https://doi:10.1520/ MPC20160031.

[11] B.B.Wu, Z.Q.Wang Y.S. Yu, X.L. Wang, C.J. Shang, and R.D.K. Misra, Thermodynamic basis of twin-related variant pair in high strength low alloy steel, Scripta Mater., 2019, vol. 170, pp.
43-47. https://doi.org/10.1016/j.scriptamat. 2019.05.016

[12] H. Zhao and E. J. Palmiere, Influence of cooling rate on the grain-refining effect of austenite deformation in a HSLA steel, Mater.

Characterization, 2019, vol.158, art. 109990. https:/ /doi.org/10.1016/j.matchar.2019.109990

[13] J.Yin, L. Leach, M. Hillert, and A. Borgenstam, C-curves for lengthening of Widmansätten and bainitic ferrite Metal., Mater. Trans. A, 2017, vol.48A, pp. 3997-4005. https://doi.org/10.1007/ s11661-017-4196-5

[14] G. Kurdjumov and Z. Sachs, Uber den mechanismus der stahlhartung, Zeitschrift für Physik, 1930, vol. 64, no. 4-6, pp. 325-343. https:// doi.org/10.1007/BF01397346

[15] CZ. Nishiyama, Lattice distortion and atomic displacements during the fcc/bcc martensitic transformation Scientific Reports of Tohoku Imperial University, 1934, vol.23 pp. 637-644.

[16] G. Wassermann, Einfluss der á- ă-umwandlung eines irreversiblen nickelstahls auf kristallorientierung und zugfestigkeit Archiv Eisenhuttenwesen, 1933, vol. 6, pp. 347-351.

[17] S. Morito, H. Tanaka, R. Konishi, T. Furuhara, and T. Maki, The morphology and crystallography of lath martensite in Fe-C alloys, Acta Mater., 2003, vol.51, no. 6, pp. 1789-1799. https://doi.org/ 10.1016/S1359-6454(02)00577-3

[18] S. Morito, X. Huang, T. Furuhara, T. Maki, and N. Hansen, The morphology and crystallography of lath martensite in alloy steels, Acta Mater., 2006, vol.54, no. 19, pp. 53235331. https://doi.org/10.1016/j.actamat.2006.07.009

[19] HKDH. Bhadeshia, Bainite in steels (London, The Institute of Materials, 1992).

[20] L. Malet, M.R. Barnett, P.J. Jacques, and S. Godet, Variant selection during the -to-a phase transformation in hot-rolled bainitic TRIP-aided steels, Scr. Mater., 2009, vol. 61, no. 5, pp. 520-523. https://doi.org/10.1016/j.scriptamat.2009.05.015

[21] Sh. Zhang, Sh. Morito, and Yu. Komizo, Variant selection of low carbon high alloy steel in an austenite grain during martensite transformation, ISIJ International, 2012, vol. 52, no. 3, pp. 510-515. https://doi.org/10.2355/ isijinternational.52.510

[22] N. Takayama, G. Miyamoto, and T. Furuhara, Effects of transformation temperature on variant pairing of bainitic ferrite in low carbon steel, Acta Mater., 2012, vol. 60, no. 5, pp. 2387-2396. https://doi.org/10.1016/j.actamat.2011.12.018

[23] G. Miyamoto, N. Iwata, N. Takayama, T. Furuhara, Quantitative analysis of variant selection in 
ausformed lath martensite, Acta Mater., 2012, vol. 60, no. 3, pp. 1139-1148. https://doi.org/ 10.1016/j.actamat.2011.11.018

[24] A. B. Greninger and A. R. Troyano, The mechanism of martensite formation, Metals Transactions, 1949, vol.185, pp. 590-598.

[25] E. C. Bain, The nature of martensite, Transactions of AIME, 1924, vol.70, pp. 25-46.

[26] A. Stormvinter, G. Miyamoto, T. Furuhara, P. Hedström, and A. Borgenstam, Effect of carbon content on variant pairing of martensite in $\mathrm{Fe}-\mathrm{C}$ alloys, Acta Mater., 2012, vol. 60, no. 20, pp. 7265-7274. https://doi.org/10.1016/j.actamat. 2012.09.046

[27] H. Beladi, Y. Adachi,I. Timokhina, and P.D. Hodgson, Crystallographic analysis of nanobainitic steels, Scr. Mater., 2009, vol. 60, no. 6, pp. 455-458. https://doi.org/10.1016/ j.scriptamat.2008.11.030

[28] C. Cayron, EBSD imaging of orientation relationships and variant groupings in different martensitic alloys and Widmanstätten iron meteorites, Mater. Charact., 2014, vol. 94, pp. 93-110. https://doi.org/10.1016/j.matchar. 2014.05.015

[29] H. Terasaki, Y. Shintome, A. Takada, Y.I. Komizo, K. Moriguchi, and Y. Tomio, Visualization and analysis of variant grouping in continuously cooled low-carbon steel welds, Metall. Mater. Trans. A, 2014, vol. 45A, pp. 3554-3559. https:// doi.org/10.1007/s11661-014-2268-3

[30] N.Y. Zolotorevskii, A. Zisman, S. Panpurin, Y.F. Titovets, S. Golosienko, and E. Khlusova, Effect of the grain size and deformation substructure of austenite on the crystal geometry of bainite and martensite in low-carbon steels, Met. Sci. Heat Treat., 2014, vol.55, pp. 550-558. https://doi.org/ 10.1007/s11041-014-9668-2

[31] N. Bernier, L. Bracke, L. Malet, and S. Godet, Crystallographic reconstruction study of the effects of finish rolling temperature on the variant selection during bainite transformation in C-Mn High-strength steels, Metall. Mater. Trans. A, 2014, vol. 45A, pp. 5937-5955. https:// doi.org/10.1007/s11661-014-2553-1

[32] M.X. Zhang and P.M. Kelly, Accurate orientation relationship between ferrite and austenite in low carbon martensite and granular bainite, Scr. Mater., 2002, vol. 47, no. 11, pp. 749-755. https://doi.org/10.1016/s1359-6462(02)00196-3

[33] G. Nolze, Characterization of the fcc/bcc orientation relationship by EBSD using pole figures and variants, Z. Metallkd., 2004, vol. 95, no 9, pp. 744-755. https://doi.org/10.3139/ 146.018017

[34] G. Nolze, Improved determination offcc/bcc orientation relationships by use of high-indexed pole figures, Cryst. Res. Technol., 2006, vol. 41, no. 1, pp. 72-77. https://doi.org/10.1002/ crat. 200410533

[35] C. Cayron, B. Artaud, and L. Briottet, Reconstruction of parent grains from EBSD data, Mater Charact., 2006, vol.57, no. 4-5, pp. 386-401. https://doi.org/10.1016/j.matchar. 2,006.03.008

[36] G. Miyamoto, N. Takayama, and T. Furuhara, Accurate measurement of the orientation relationship of lath martensite and bainite by electron backscatter diffraction analysis, Scr. Mater., 2009, vol. 60, no. 12, pp. 1113-1116. https://doi.org/10.1016/j.scriptamat.2009.02.053

[37] G .Miyamoto, N. Iwata, N. Takayama, and T. Furuhara, Mapping the parent austenite orientation reconstructed from the orientation of martensite by EBSD and its application to ausformed martensite, Acta Mater., 2010, vol. 58, no. 19, pp. 6393-6403. https://doi.org/10.1016/ j.actamat.2010.08.001

[38] M. Humbert, P. Blaineau, L. Germain, and N. Gey, Refinement of orientation relations occurring in phase transformation based on considering only the orientations of the variants, Scr. Mater., 2011, vol.64, no. 12, pp. 114-117. https://doi.org/ 10.1016/j.scriptamat.2010.09.016

[39] N. Bernier, L. Bracke, L. Malet, and S. Godet, An alternative to the crystallographic reconstruction of austenite in steels, Mater. Charact., 2014, vol.89, pp. 23-32. https://doi.org/ 10.1016/j.matchar.2013.12.014

[40] M. Humbert, L. Germain, N. Gey, and E. Boucard, Evaluation of the orientation relations from misorientation between inherited variants: application to ausformed martensite, Acta Mater., 2015, vol. 82, pp. 137-144. https://doi.org/ 10.1016/j.actamat.2014.09.007

[41] N.Y. Zolotorevsky, S.N. Panpurin, A.A. Zisman, and S.N. Petrov, Effect of ausforming and cooling condition on the orientation relationship in martensite and bainite of low carbon steels, Mater. Charact., 2015, vol. 107, pp. 278-282. http:// doi.org/10.1016/j.matchar.2015.07.023

[42] E. Gomes and L.A.I. Kestens, Fully automated orientation relationship calculation and prior austenite reconstruction by random walk clustering, IOP Conf. Ser. Mater. Sci. Eng., 2015, 
vol. 82, pp. 24-29. https://doi.org/10.1088/1757899X/82/1/012059

[43] T. Nyyssönen, M. Isakov, P. Peura, and V.-T. Kuokkala, Iterative determination of the orientation relationship between austenite and martensite from a large amount of grain pair misorientations, Metall. Mater. Trans. A, 2016, vol. 47A, pp. 2587-2590. https://doi.org/10.1007/ s11661-016-3462-2

[44] A.F. Brust, E.J. Payton, V. Sinha, V.A. Yardley, and S.R. Niezgoda, Characterization of martensite orientation relationships in steels and ferrous alloys from EBSD data using bayesian inference, Metall. Mater. Trans. A, 2020, vol.51, pp. 142-153. https://doi.org/10.1007/s11661-019-05514-4

[45] L. Germain, N. Gey, R. Mercier, P. Blaineau, and M. Humbert, An advanced approach to reconstructing parent orientation maps in the case of approximate orientation relations: Application to steels, Acta Mater., 2012, vol. 60, no. 11, pp. 4551-4562. https://doi.org/10.1016/ j.actamat.2012.04.034

[46] L. Morsdorf, C.C. Tasan , D. Ponge, and D. Raabe, $3 D$ structural and atomic-scale analysis of lath martensite: Effect of the transformation sequence, Acta Mater., 2015, vol. 95, pp. 366-377. http://doi.org/10.1016/j.actamat.2015.05.023

[47] M. S. Wechsler, D. S. Lieberman, and T. A. Read, On the theory of the formation of martensite, Transactions of AIME, 1953, vol. 197, pp. 15031515.

[48] J.S. Bowels and J. K. Mackenzie, The crystallography of marensite transformations III. Face-centered cubic to body-centered tetragonal transformations, Acta Metall., 1954, vol. 2, pp. 224-234.

[49] R. Bullough and B.A. Bilby, Continuous distribution of dislocations: Surface dislocations and the crystallography of martensitic transformation, Proceedings of the Royal Society of London B, 1956, vol. 69, pp. 1276-1286.

[50] G. Miyamoto, A. Shibata, T. Maki, and T. Furuhara, Precise measurement of strain accommodation in austenite matrix surrounding martensite in ferrous alloys by electron backscatter diffraction analysis, Acta Mater., 2009, vol. 57, no. 4, pp. 1120-1131. https://doi.org/ 10.1016/j.actamat.2008.10.050

[51] T. Hayashi, S. Morito, and T. Ohba, Local distribution of orientation relationship and microstructure evolution of lath martensite in an ultra-low-carbon steel, Scr. Mater., 2020, vol. 180, pp. 1-5. https://doi.org/10.1016/j.scriptamat. 2020.01.011

[52] T. Moritani, N. Miyajima, T. Furuhara, and T. Maki, Comparison of interphase boundary structure between bainite and martensite in steel, Scri. Mater., 2002, vol. 47, no. 3, pp. 193-199. https://doi.org/10.1016/S1359-6462(02)00128-8

[53] B.P. Sandvik and C.M. Wayman, Characteristics of lath martensite: Part I. Crystallographic and substructural features, Metall. Trans. A, 1983, vol. 14A, no, 4 pp. 809-822. https://doi.org/ $10.1007 / \mathrm{bf0} 2644284$

[54] G.J. Mahon, J.M. Howe, and S. Mahajan, HRTEM study of the $\{252\}_{\gamma}$ austenite-martensite interface in an Fe-8Cr-1C alloy, Phil. Mag. Letters, 1989, vol. 59, no. 6, pp. 273-279. https:// doi.org/10.1080/09500838908206355

[55] H.K.D.H. Bhadeshia, and R. Honeycombe, Steel microstructure and properties (Amsterdam, Elsevier, 2006).

[56] P.M. Kelly, Crystallography of martensite transformations in steels In: Phase transformations in steels, V.2: Diffusionless transformations, high strength steels, modeling and advanced analytical techniques, ed. by E. Pereloma and D.V. Edmonds (Oxford, Woodhead Publishing, 2012), p. 3-33.

[57] D. Dunne, Martensite, Metals, 2018, vol. 8, no. 6, pp. 395-1 - 395-17. https://doi.org/10.3390/ met8060395

[58] N. D. H. Ross and A. G. Crocker, A generalized theory of martensite crystallography and its applications to transformations in steels, Acta Metall., 1970, vol. 18, no. 4, pp. 405-418. https:// doi.org/10.1016/0001-6160(70)90126-4

[59] P.M. Kelly, Crystallography of lath martensite in steels, Mater. Transactions JIM, 1992, vol. 33, no. 2, pp. 235-242. https://doi.org/10.2320/ matertrans 1989.33.235

[60] L. Qi, A.G. Khachaturyan, and J. W. Morris, The microstructure of dislocated martensite steel: Theory, Acta Mater., 2014, vol.76, pp. 23-35. http://doi.org/10.1016/j.actamat.2014.04.038

[61] J. Ball and R. James, Fine phase mixtures as minimizer of energy, Archives for Rational Mechanics and Analysis, 1987, vol.100, pp. 13-52. https://doi.org/10.1007/BF00281246

[62] K. Koumatos and A. Muehlemann, The morphology of lath martensite: A new perspective, MATEC Web of Conferences, 2015, vol. 33, pp. 07003-1 - 07003-8. https://doi.org/ 10.1051/matecconf/20153307003

[63] A. P. Baur, C. Cayron and R. E. Logé, $\{225\} \breve{a}$ habit planes in martensitic steels: from the 
PTMC to a continuous model, Scientific Reports, 2017, vol. 7, art. 40938. https://doi.org/10.1038/ srep40938

[64] C. Cayron, A. Baur, and R. E. Logé, Intricate morphologies of laths and blocks in low-carbon martensitic steels, Materials and Design, 2018, vol. 54, pp. 81-95. https://doi.org/10.1016/ j.matdes.2018.05.033

[65] M. Petersmann, T. Antretter, G. Cailletaud, A. Sannikov, U. Ehlenbröker, and F.D. Fischer, Unification of the non-linear geometric transformation theory of martensite and crystal plasticity - Application to dislocated lath martensite in steels, International Journal of Plasticity, 2019, vol. 119, pp. 140-155. https:// doi.org/10.1016/j.ijplas.2019.02.016

[66] F. Archie, M. Z. Mughal, M. Sebastiani,

E. Bemporad. and S. Zaefferer, Anisotropic distribution of the micro residual stresses in lath martensite revealed by FIB ring-core milling technique, Acta Materialia, 2018, vol. 150, pp. 327-338. https://doi.org/10.1016/ j.actamat.2018.03.030

[67] J.D. Eshelby, The determination of the elastic field of an ellipsoidal inclusion and related problems, Proceedings of the Royal Society of London A, 1957. vol. 241, pp. 376-396.
[68] A. Zisman, Predictive micromechanical model for plastic accommodation and crystallography of martensite embryo, International Journal of Engineering Science, 2020, vol. 150, art. 103245. https://doi.org/10.1016/j.ijengsci.2020.103245

[69] G.B. Olson, Fine structure of interphase boundaries, Acta Mater., 1981, vol. 29, no. 8, pp. 1475-1484. https://doi.org/10.1016/00016160(81)90182-6

[70] R.C. Pond, X. Ma, and J.P. Hirth, A dislocation model of parent-martensite interfaces, In: Proceedings of ICOMAT-08, ed. by G.B. Olson, D.S. Lieberman, and A. Saxens, 2009, pp.107-114.

[71] J.M. Howe, R.C. Pond. and J.P. Hirth, The role of disconnections in phase transformations, Progr. in Mater. Sci., 2009, vol.54, no. 6, pp. 792-838. https://doi.org/10.1016/j.pmatsci.2009.04.001

[72] PM. Kelly, The phenomenological theory of martensite crystallography (PTMC) versus the topological model (TM), In: Proceedings of ICOMAT-08, ed. by G.B. Olson, D.S. Lieberman, and A. Saxens, 2009, p.115-121.

[73] J.P. Hirth and R.C. Pond, Response to "The phenomenological theory of martensite crystallography (PTMC) versus the topological model (TM) “, In: Proceedings of ICOMAT-08, ed. by G.B. Olson, D.S. Lieberman, and A. Saxens, 2009, p.123-128. 